\title{
Resource Management and 3D Deployment in Power-Efficient Multi-UAV Networks
}

\author{
Yongjun Sun ( $\nabla$ yjsun@mail.xidian.edu.cn ) \\ Xidian University https://orcid.org/0000-0002-1145-1750
}

\section{Liaoping Zhang}

Xidian University

\section{Zujun Liu}

Xidian University

\section{Research Article}

Keywords: multi-UAV networks, resource management, 3D deployment, load balance, power efficient Posted Date: December 8th, 2021

DOI: https://doi.org/10.21203/rs.3.rs-1051789/v1

License: (c) (1) This work is licensed under a Creative Commons Attribution 4.0 International License. Read Full License 


\title{
Resource Management and 3D Deployment in Power-Efficient multi-UAV Networks
}

\author{
Yongjun Sun, Liaoping Zhang, and Zujun Liu
}

\author{
School of telecommunication engineering, Xidian University, Xi'an, China
}

\begin{abstract}
In this paper, the scenario in which multiple unmanned aerial vehicles (UAVs) provide service to ground users is considered. Under the condition of satisfying the minimum rate per user and system load balance, the user association, bandwidth allocation and three dimensional (3D) deployment of multi-UAV networks are optimized jointly to minimize the total downlink transmit power of UAVs. Since the problem is hard to solve directly, it is decomposed into three sub-problems, and then the problem is solved by alternating iteration algorithm. First, when the UAV's location is determined, a modified $K$-means algorithm is used to obtain balanced user clustering. Then, when the user association and UAV's 3D deployment are determined, the convex optimization method is used to obtain the optimal bandwidth allocation. Finally, when the user association and optimal bandwidth allocation are determined, a modified differential evolution algorithm is proposed to optimize the 3D location of the UAVs. Simulation results show that the proposed algorithm can effectively reduce the transmit power of UAVs compared with the existing algorithms under the conditions of satisfying the minimum rate of ground users and system load balance.
\end{abstract}

Index Terms -multi-UAV networks, resource management, 3D deployment, load balance, power efficient

\section{INTRODUCTION}

$\mathrm{W}$ ITH the rapid development of mobile communication technology and Unmanned Aerial Vehicle (UAV) industry, the application of Unmanned Aerial Vehicle in the field of wireless communication has become a research hotspot [1][2]. UAVs can be used as aerial Base Station (BS) or aerial mobile relay, which has advantages in emergency or temporary communication scenarios. For example, in case of terrestrial BSs failure, UAV-BSs can be rapidly deployed to meet the demand for wireless services.

Different from the traditional base station on the ground, UAV base station has some advantages, such as fast and flexible on-demand deployment, better line-of-sight (LoS) communications link, and high mobility in the three dimensional (3D) space. UAVs can flexibly adjust their locations according to the location distribution and requirement of users, which further enhances the performance of the communication networks.

However, due to the limited on-board energy of UAV, energy consumption is required not only for hovering attitude, but also for communication process. Reducing energy consumption can improve energy efficiency and increase UAV service time, so how to reduce the energy consumption of UAV is the key to design UAV-enabled communication system. In addition, for multi-UAV system, load balance is also a key factor because of the limited resources of each UAV.

In [3], the user association between UAVs and ground users is determined by using K-means algorithm. Then, the horizontal location of UAV is optimized to minimize the total minimum transmit power of UAVs under the constraint of minimum transmit delay. The closed expressions of the optimal location in two special case are obtained, and a machine learning algorithm is proposed to obtain a suboptimal $2 \mathrm{D}$ horizontal location. In [4], a successive convex approximation (SCA) method is used to optimize the $3 \mathrm{D}$ deployment of single UAV to minimize the average transmit power. In [5], the particle swarm optimization (PSO) is used to optimize 3D deployment in single UAV scene to minimize transmit power. Mozaffari Mohammad et al obtained the suboptimal UAV horizontal 2D location by linear approximation of the minimum transmit power [6][7]. The authors in [8] mainly studies the problem of UAV downlink transmit power minimization from the perspective of UAV height control.

To solve the load balance problem in multi-UAV systems, an improved K-means clustering algorithm was proposed to minimize the uplink-transmit power of devices of Internet of Things (IoT) and UAV is associated with a nearly equal number of IoT devices [9]. In [10], user association and UAV location were optimized jointly to minimize the maximum UAV traffic load. Authors in [11] studied the load balance when users are non-uniformly distributed and proposed the user partition algorithm based on the optimal transport theory to maximize the average data service. In order to balance the load between UAVs and the ground base station, a many-to-one matching game was proposed to maximize the network utility [12]. A multi-UAV load balance deployment algorithm based on differential evolution algorithm was proposed to minimize the time delay of UAV to IoT devices [13]. However, the works mentioned above do not consider jointly the problem of $3 \mathrm{D}$ deployment and bandwidth allocation with load balance constraints.

In this paper, user association, bandwidth allocation and UAV's 3D deployment are optimized jointly to minimize total UAVs' downlink transmit power. Due the problem is hard to solve directly, it is decomposed into three sub-problems to solve alternately. When the UAV's 3D location is determined, 
a modified K-means algorithm is used to obtain balanced user association. When the user association and UAV's 3D location are determined, the convex optimization method is adopted to obtain the optimal bandwidth allocation. When user association and bandwidth allocation are determined, a modified differential evolution algorithm (MDE) is proposed to optimize the 3D location of UAVs.

\section{SYSTEM MODEL AND PROBLEM FORMULATION}

\section{A. System Model}

As shown in Fig.1, We study the downlink communication between ground users and UAVs. There are $K$ ground users randomly distributed in a square area and $M$ UAV-BSs provide communication services, whose index sets are denoted as $\mathcal{K}=\{1,2, \ldots, K\}$ and $\mathcal{M}=\{1,2, \ldots, M\}$, respectively. Note that the locations of the $K$ users are static and prior known by a control center. The location of $\mathrm{UAV}_{m} m \in \mathcal{M}$ is given by $\boldsymbol{v}_{\mathrm{m}}=\left(X_{m}, Y_{m}, H_{m}\right)$ and the location of ground user $k \in \mathcal{K}$ is given by $\boldsymbol{x}_{\mathbf{k}}=\left(x_{k}, y_{k}, 0\right)$. In the model, UAVs transmit on different frequency bands, so there is no interference between UAVs. In order to avoid interference between users in the same UAV cell, UAV adopts frequency division multiple access technology to serve the ground users. Each UAV allocates its available frequency band to associated users to satisfy the demand of each user.

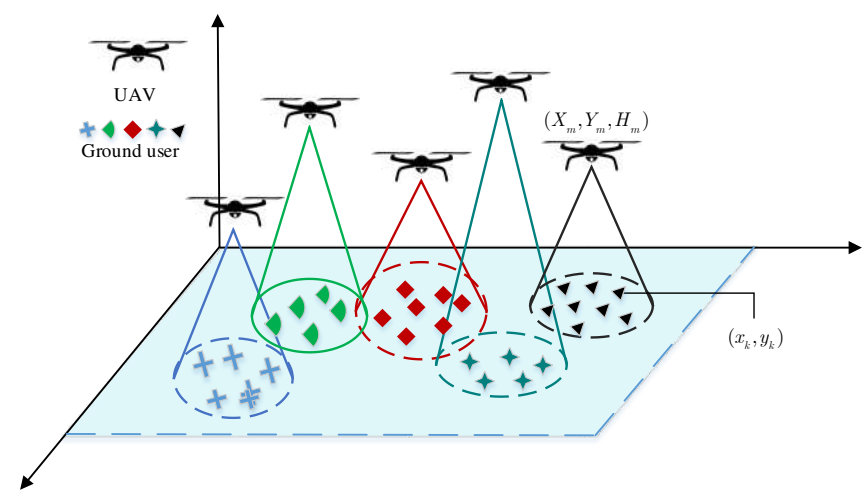

Fig. 1. Multi-UAV serve multi-user system

\section{B. Air-to-Ground Channel Model}

In the UAV air-to-ground (ATG) communication, the typical channel model is the probability line-of-sight model, which is the probability weighted combination of line-of-sight (LoS) and non-line-of-sight (NLoS) [14][15]. The LoS and NLoS path losses between $\mathrm{UAV}_{m}$ and ground user $k$ are expressed as

$$
\begin{aligned}
L_{m, k}^{L o S} & =\left(\frac{4 \pi f_{c} d_{m, k}}{c}\right)^{\alpha} \eta_{L o S} \\
L_{m, k}^{N L O S} & =\left(\frac{4 \pi f_{c} d_{m, k}}{c}\right)^{\alpha} \eta_{N L o S}
\end{aligned}
$$

where $f_{\mathrm{c}}$ is the carrier frequency, $\mathrm{c}$ is the speed of light, $\eta_{L o S}$ and $\eta_{\mathrm{NLoS}}$ are the losses for the LoS and NLoS links due to the free space propagation respectively. $\alpha$ is the path loss exponent. The distance between $\mathrm{UAV}_{m}$ and ground user $k$ is

$$
d_{m, k}=\sqrt{\left(X_{m}-x_{k}\right)^{2}+\left(Y_{m}-y_{k}\right)^{2}+H_{m}^{2}}
$$

In the ATG channel model, LoS link is given probabilistically due to the uncertainty of possible obstacles (such as tall buildings, trees and mountains) in the networks, and the probability of LoS link depends on the location of the UAV and the ground user, network environment and elevation angle from users to UAVs. The LoS probability is given by

$$
P_{m, k}^{L o S}=\frac{1}{1+\varphi_{1} \exp \left[-\varphi_{2}\left(\theta_{m, k}-\varphi_{1}\right)\right]}
$$

where $\varphi_{1}$ and $\varphi_{2}$ are constant values, which depend on the network environment. $\theta_{m, k}=\frac{180}{\pi} \sin ^{-1}\left(\frac{H_{m}}{d_{m, k}}\right)$ (in degree) is the elevation angle from the user $k$ to $\mathrm{UAV}_{m}$.

The NLoS probability is

$$
P_{m, k}^{N L o S}=1-P_{m, k}^{L o S}
$$

Hence, the mean probabilistic path loss of the air-to-ground link is the averaged value of LoS and NLoS links, which is given by

$$
\begin{aligned}
& \bar{L}_{m, k}=P_{m, k}^{L O S} L_{m, k}^{L O S}+P_{m, k}^{N L o S} L_{m, k}^{N L o S} \\
& =P_{m, k}^{\operatorname{LoS}}\left(\frac{4 \pi f_{c} d_{m, k}}{c}\right)^{\alpha} \eta_{L o S}+P_{m, k}^{N L O S}\left(\frac{4 \pi f_{c} d_{m, k}}{c}\right)^{\alpha} \eta_{N L o S} \\
& =\left[P_{m, k}^{L O S} \eta_{L o S}+P_{m, k}^{N L o S} \eta_{N L o S}\right]\left(\frac{4 \pi f_{c} d_{m, k}}{c}\right)^{\alpha}
\end{aligned}
$$

The average channel gain between $\mathrm{UAV}_{m}$ and user $k$ is $g_{m, k}=\frac{1}{\bar{L}_{m, k}}$.

\section{Problem Formulation}

According to Shannon formula, the achievable rate of ground user $k$ from $\mathrm{UAV}_{m}$ is

$$
R_{m, k}=w_{m, k} \log _{2}\left(1+\frac{p_{m, k} g_{m, k}}{n_{0} w_{m, k}}\right)
$$

where $w_{m, k}$ is the bandwidth allocated by $\mathrm{UAV}_{m}$ to user $k, p_{m, k}$ is the transmit power from $\mathrm{UAV}_{m}$ to user $k$, and $n_{0}$ is the noise power spectral density.

Define a user association indicator $a_{m, k}$, which is a binary variable. When user $k$ is served by $\mathrm{UAV}_{m}, a_{m, k}=1$, otherwise, $a_{m, k}=0, k \notin S_{m}$, where $S_{m}$ is the set of users served by $\mathrm{UAV}_{m}$. Both $S_{m}$ and $a_{m, k}$ in the following paper represent the user association status. Assuming that the minimum rate requirement of ground user $k$ is $R_{k}^{\min }$, then $R_{m, k} \geq R_{k}^{\min }$. In order to save the energy of UAV, we suppose 
that the UAV always transmits data which satisfies the minimum data requirement, that is, $R_{m, k}=R_{k}^{\min }$. Therefore, the minimum transmit power required by $\mathrm{UAV}_{m}$ to satisfy the rate requirement of its associated user $k$ is

$$
p_{m, k}^{\min }=\left(2^{R_{k}^{\min } / w_{m, k}}-1\right) n_{0} w_{m, k} \bar{L}_{m, k}
$$

Therefore, the total transmit power required by $\mathrm{UAV}_{m}$ is $p_{m}=\sum_{k \in S_{m}} p_{m, k}^{\min }$. From the expression, it can be seen that the transmit power required by each UAV is related to associated user set $S_{m}$, bandwidth allocation $w_{m, k}$ and average path loss $\bar{L}_{m, k}$, while $\bar{L}_{m, k}$ is related to UAV's $3 \mathrm{D}$ location and LoS probability.

Our purpose is to optimize user association, bandwidth allocation and 3D location of UAV to minimize the total transmit power of UAV, while ensuring the system load balance and the minimum rate demand of each user. Mathematically, the sum power minimization problem is

$$
\begin{gathered}
\min _{\left\{w_{m, k}\right\},\left\{S_{m}\right\},\left\{v_{\mathrm{m}}\right\}} \sum_{m=1}^{M} p_{m}=\sum_{m=1}^{M} \sum_{k \in S_{m}}\left(2^{R_{k}^{\min } / w_{m, k}}-1\right) n_{0} w_{m, k} \bar{L}_{m, k} \\
\text { s.t. } \sum_{k \in S_{m}} w_{m, k} \leq B_{m}, \forall m \in \mathcal{M} \\
w_{m, k} \geq 0, \forall m \in \mathcal{M}, k \in \mathcal{K} \\
S_{m} \cap S_{m^{\prime}}=\varnothing, \forall m, m^{\prime} \in \mathcal{M}, m \neq m^{\prime} \\
\sum_{m=1}^{M}\left|S_{m}\right|=K \\
\left|\frac{K}{M}\right| \leq\left|S_{m}\right| \leq\left|\frac{K}{M}\right| \\
\mathrm{X}_{\min } \leq X_{m} \leq \mathrm{X}_{\max } \\
\mathrm{Y}_{\min } \leq Y_{m} \leq \mathrm{Y}_{\max } \\
\mathrm{H}_{\min } \leq H_{m} \leq \mathrm{H}_{\max }
\end{gathered}
$$

where $\left\{w_{m, k}\right\}$ denotes the bandwidth allocation of $\mathrm{UAV}_{m}$, $\left\{S_{m}\right\}$ is the user association, and $\left|S_{m}\right|$ is the number of users served by $\mathrm{UAV}_{m} . \boldsymbol{v}_{\mathrm{m}}$ is the $3 \mathrm{D}$ location of $\mathrm{UAV}_{m}, B_{\mathrm{m}}$ is the total available bandwidth of $\mathrm{UAV}_{m}$. The constraints (9b) and (9c) indicate that the bandwidth allocated to users cannot exceed the total available bandwidth. The constraint (9d) indicates that each user is only associated with one UAV, and the constraint (9e) ensures that all users in the network can be served. The constraint (9f) is the load balance, which ensures that the number of associated users of each UAV is near equal. The constraints $(9 \mathrm{~g}),(9 \mathrm{~h})$ and $(9 \mathrm{i})$ indicate the 3D location constraint of the UAVs, and the subscripts $(\cdot)_{\min }$ and $(\cdot)_{\max }$ represent the boundary values of $X_{\mathrm{m}}, Y_{\mathrm{m}}$ and $H_{\mathrm{m}}$.

It is obvious that the optimization problem in (9) is a mixedinteger nonlinear programming (MINLP) problem because it contains both continuous and binary variables and the objective function is nonconvex, which is a NP-hard problem, and it is difficult to obtain the global optimal solution in polynomial time. In the next section, we will resort alternating iterative optimization (also known as block gradient descent method) to solve the problem. We propose an iterative algorithm to solve the joint optimization problem in (9) by decomposing it into three sub-problems: 1) balanced user association; 2) optimal bandwidth allocation; 3) UAV's 3D deployment. After solving these three sub-problems alternately, an iterative algorithm is used to achieve the final solution of problem in (9).

\section{PROPOSED ALGORITHM}

\section{A. Balanced Users Association}

Define $\boldsymbol{v}_{m}^{2 D}=\left(X_{m}, Y_{m}\right)$ and $\boldsymbol{x}_{k}^{2 D}=\left(x_{k}, y_{k}\right)$ as the projection location of UAVs and users on the horizontal plane respectively. Let $r_{m, k}^{2}=\left\|\boldsymbol{v}_{m}^{2 D}-\boldsymbol{x}_{k}^{2 D}\right\|^{2}$. Given the bandwidth allocation and UAV's 3D deployment, the sub-problem of balancing user association is transformed as

$$
\begin{gathered}
\min _{\left\{a_{m, k}\right\}} \sum_{m=1}^{M} \sum_{k=1}^{K} a_{m, k} r_{m . k}^{2} \\
\text { s.t.S } S_{m} \cap S_{m^{\prime}}=\varnothing, \forall m, m^{\prime} \in \mathcal{M}, m \neq m^{\prime} \\
\sum_{m=1}^{M}\left|S_{m}\right|=K \\
\left.\mid \frac{K}{M}\right\rfloor \leq\left|S_{m}\right| \leq\left\lceil\frac{K}{M}\right\rceil, \forall m \in \mathcal{M}
\end{gathered}
$$

Since the original K-means algorithm does not consider user balanced clustering, balanced K-means proposed in [16] is adopted to solve this problem. As shown in Algorithm 1, firstly, an initial center $C_{0}$ is obtained by using K-means++ algorithm, then a cost function $C_{K \times K}=\left\|\boldsymbol{x}_{j}^{2 D}-\boldsymbol{v}_{\bmod (i, M)+1}^{2 D}\right\|^{2}$, $1 \leq i, j \leq K$ is established, and finally Hungarian algorithm is used for user allocation.

\begin{tabular}{l}
\hline Algorithm 1: Balanced User Association \\
Input: Data of all users' location $\left\{\boldsymbol{x}_{\mathbf{k}}\right\}$, the number of \\
UAVs $M$. \\
Output: Users are evenly divided into $M$ clusters \\
1: $t=0$, Initialize $M$ centers $C_{0}$ using the K-means++ \\
algorithm. \\
2: Repeat \\
3: Assignment step: build the cost function $C_{K \times K}$, solve \\
4: $\quad$ Update step: Calculate new centroid locations. \\
$\quad C_{t+1}=\frac{1}{\left|S_{m}\right|} \sum_{k \in S_{m}} \boldsymbol{x}_{k}^{2 D}$. \\
5: $t=t+1$ \\
6: Until centroid locations do not change. \\
\hline \hline
\end{tabular}




\section{B. 3D Deployment of UAVs based on Modified Differential Evolution Algorithm}

Given user association and bandwidth allocation, due to UAVs can move freely in 3D space, its 3D location can be updated according to associated user locations to minimize UAVs transmit power. Therefore, the 3D deployment optimization sub-problem is expressed as:

$$
\begin{gathered}
\min _{\left\{v_{\mathrm{m}}\right\}} \sum_{m=1}^{M} p_{m}=\sum_{m=1}^{M} \sum_{k \in S_{m}}\left(2^{R_{k}^{\min } / w_{m, k}}-1\right) n_{0} w_{m, k} \bar{L}_{m, k} \\
\text { s.t. } X_{\min } \leq X_{m} \leq X_{\max } \\
\mathrm{Y}_{\text {min }} \leq Y_{m} \leq \mathrm{Y}_{\max } \\
\mathrm{H}_{\text {min }} \leq \mathrm{H}_{m} \leq \mathrm{H}_{\text {max }}
\end{gathered}
$$

The objective function contains $\bar{L}_{m, k}$, which is a variable related to the UAV's 3D location $\boldsymbol{v}_{m}$, and it is hard to find the optimal UAV 3D location. However, heuristic algorithm has the advantage of solving complex optimization problems, so we propose a modified differential evolution algorithm (MDE) to solve the 3D deployment problem of multi-UAV networks.

In the process of initial user association, K-means++ algorithm generates a group of UAV locations. However, Kmeans++ aims to minimize the Euclidean distance on the horizontal plane. On the one hand, from the equations (3) and (4), it can be seen that the minimum power is not only related to the Euclidean distance from the UAVs to the users, but also related to the probability of LoS link. On the other hand, we need to consider the influence of UAVs' height, so the UAV location generated by $\mathrm{K}$-means++ is not optimal, and 3D deployment of UAVs still need to be improved.

Differential evolution algorithm is a simple but powerful heuristic algorithm, which can search and solve a problem directly through differential evolution operation. In this paper, a modified differential evolution algorithm is proposed to solve the 3D deployment problem to minimize the transmit power of UAVs.

In the traditional differential evolution algorithm, each individual is represented as the entire UAV's deployment $\left\{\boldsymbol{v}_{1}, \boldsymbol{v}_{2}, \ldots, \boldsymbol{v}_{M}\right\}$, and then a population is formed by the randomly generated $N P$ individuals. As shown in (12), a matrix represents a population, and its size is $N P \times 3 M$. Each row of the matrix represents an individual, and each individual represents the UAV deployment.

$$
\left[\begin{array}{cccc}
\boldsymbol{v}_{1}^{(1)} & \boldsymbol{v}_{2}^{(1)} & \ldots & \boldsymbol{v}_{M}^{(1)} \\
\vdots & \vdots & \ddots & \vdots \\
\boldsymbol{v}_{1}^{(N P)} & \boldsymbol{v}_{2}^{(N P)} & \ldots & \boldsymbol{v}_{M}^{(N P)}
\end{array}\right]_{N P \times 3 M}
$$

We propose a new coding mechanism to generate population which is similar to [17]. As shown in the matrix (13), each individual is represented as the 3D location of one UAV, that is, each row of the matrix represents the 3D location of each UAV.

$$
\left[\begin{array}{ccc}
X_{1} & Y_{1} & H_{1} \\
X_{2} & Y_{2} & H_{2} \\
\vdots & \vdots & \vdots \\
X_{M} & Y_{M} & H_{M}
\end{array}\right]_{M \times 3}
$$

In this way, the dimension of each individual is fixed to 3 , which will not change with the number of UAVs.

In order to accelerate the convergence of the algorithm, the UAVs' 2D location obtained by balanced K-means algorithm and a random height is given to each UAV as the initial 3D deployment.

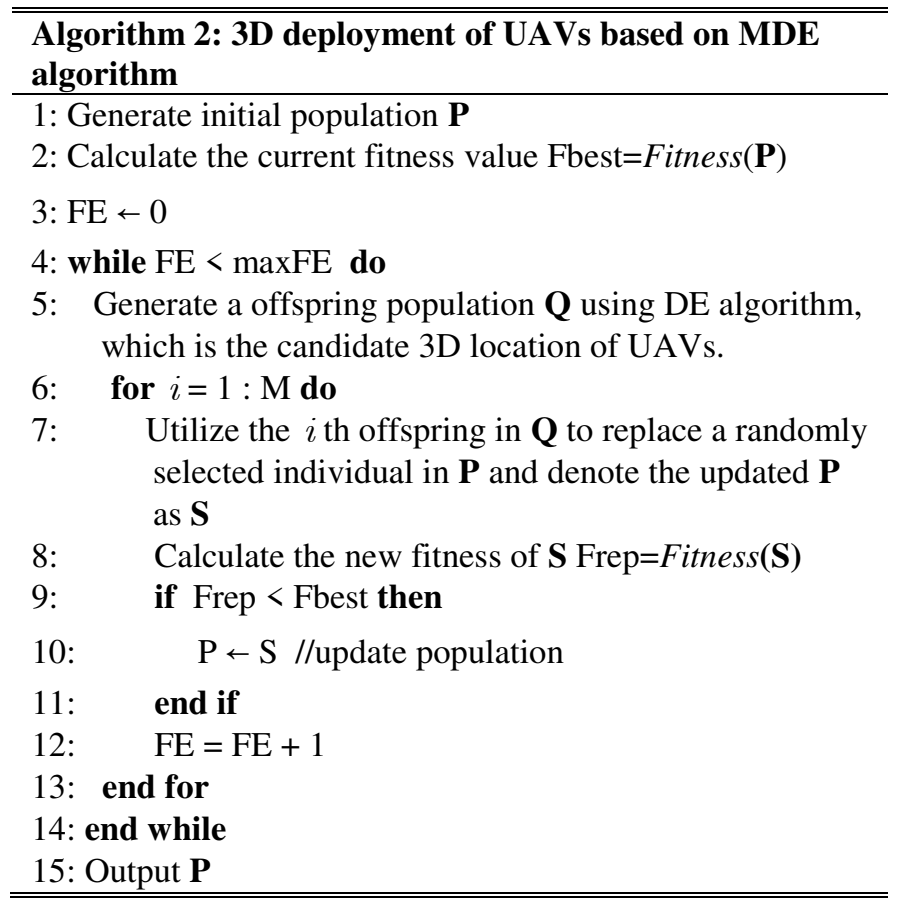

\section{Optimal Bandwidth Allocation}

Given user association and UAV's 3D deployment, each UAV will allocate its bandwidth to its associated users while minimizing the transmit power. Let $F_{k}=n_{0} w_{m, k} \bar{L}_{m, k}$. The bandwidth allocation sub-problem is converted to

$$
\begin{gathered}
\min _{\left\{w_{m, k}\right\}} \sum_{m=1}^{M} p_{m}=\sum_{m=1}^{M} \sum_{k \in S_{m}} F_{k}\left(2^{R_{k}^{\min } / w_{m, k}}-1\right) w_{m, k} \\
\text { s.t. } \sum_{k \in S_{m}} w_{m, k} \leq B_{m}, \forall m \in \mathcal{M} \\
w_{m, k} \geq 0, \forall m \in \mathcal{M}, k \in \mathcal{K}
\end{gathered}
$$

Define the function $f(x)=\left(2^{R / x}-1\right) x, x>0$. Due to $f^{\prime \prime}(x)=\frac{(R \ln 2)^{2} 2^{R / x}}{x^{3}}>0$, so function $f(x)$ is a convex function, which indicates that problem (14) is a convex problem with given $\bar{L}_{m, k}$ and $S_{m}$. The lagrangian function of convex problem (14) is 
$\mathcal{L}\left(w_{m, k}, \lambda_{k}\right)=\sum_{k \in S_{m}} F_{k} w_{m, k}\left(2^{\frac{R_{k}^{\min }}{w_{m, k}}}-1\right)+\lambda_{k}\left(\sum_{k \in S_{m}} w_{m, k}-B_{m}\right), \forall m$

where $\lambda_{k}$ is the non-negative Lagrange multiplier. According to [[18], Appendix A], the KKT conditions of (14) are

$$
\begin{gathered}
\frac{\partial \mathcal{L}}{\partial w_{m, k}}=F_{k}\left(2^{\frac{R_{k}^{\min }}{w_{m, k}}}-\frac{(\ln 2) R_{k}^{\min }}{w_{m, k}} 2^{\frac{R_{k}^{\min }}{w_{m, k}}}-1\right)+\lambda_{k}=0 \\
\lambda_{k} \geq 0 \\
\lambda_{k}\left(\sum_{k \in S_{m}} w_{m, k}-B_{m}\right)=0 \\
\sum_{k \in S_{m}} w_{m, k} \leq B_{m} \\
w_{m, k} \geq 0
\end{gathered}
$$

Define the function $u(x)=x e^{x}-e^{x}+1, x \geq 0$, Due to $u^{\prime}(x)=x e^{x}>0, \forall x>0$, Thus function $u(x)$ is strictly increasing and $u(x)>u(0)=0$. Therefore,

$$
w_{m, k}=\left[\frac{(\ln 2) R_{k}^{\min }}{u^{-1}\left(\lambda_{k} / F_{k}\right)}\right]^{+}
$$

where $u^{-1}(x)$ is the inverse function of $u(x)$. Due to $\lambda_{k}=F_{k} u\left(\frac{(\ln 2) R_{k}^{\min }}{w_{m, k}}\right)>0$, so $\sum_{k \in S_{m}} w_{m, k}=B_{m}$.we can obtain optimal bandwidth allocation by using the bisection method as shown in Algorithm 3.

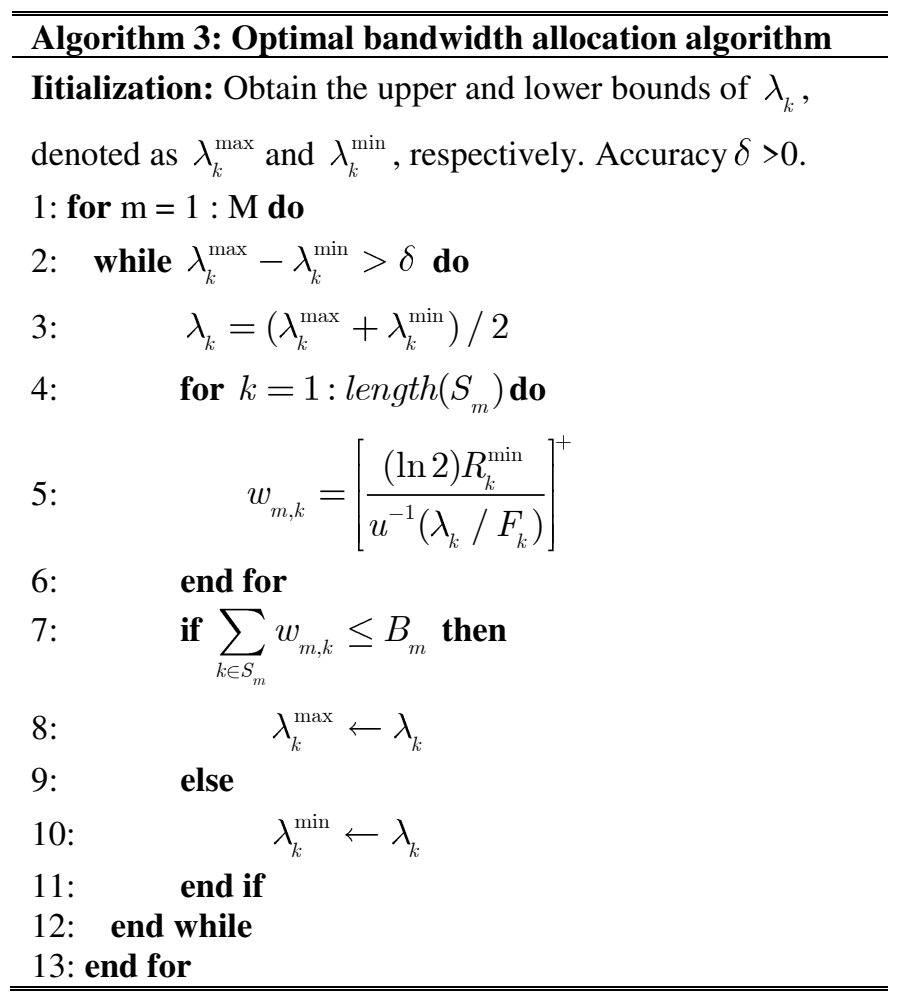

\section{Joint Iterative Optimization Algorithm}

In this section, an alternate iterative algorithm is presented in Algorithm 4 to solve the joint user association, bandwidth allocation and UAV 3D deployment problems.

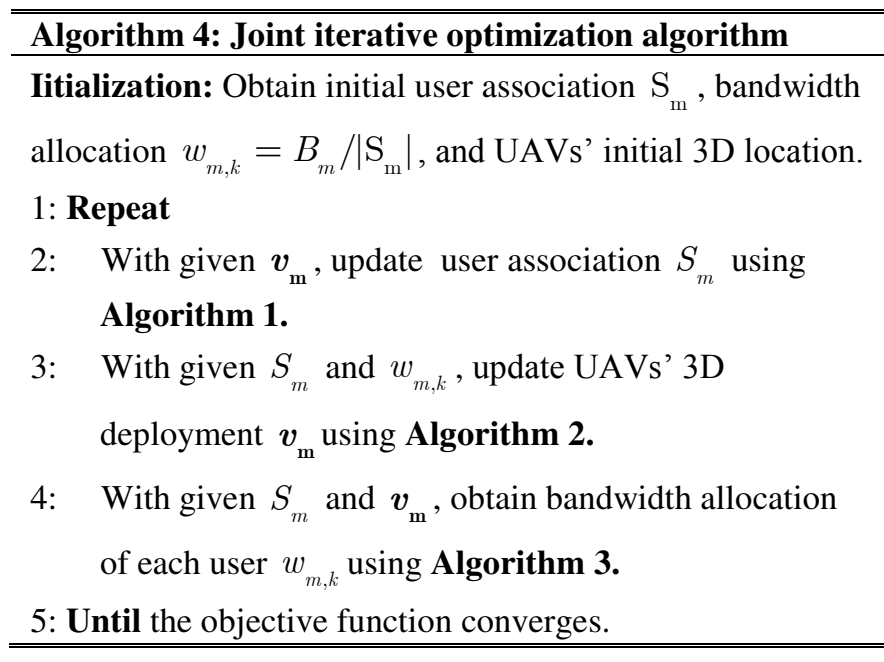

\section{Simulation RESUlts}

\section{A. Simulation Parameters Setting}

Considering that $K$ users are randomly distributed within a square area of $1000 m \times 1000 m$, and evenly served by $M$ UAVs. Other main simulation parameters are set as shown in Table I TABLE I SIMULATION PARAMETERS

\begin{tabular}{lll}
\hline \hline Symbol & Description & Value \\
\hline$f_{c}$ & Carry frequency & $2 \mathrm{GHz}$ \\
$n_{0}$ & Noise power spectral density & $-170 \mathrm{dBm} / \mathrm{Hz}$ \\
$\varphi_{1}$ & Environment parameters & 11.95 \\
$\varphi_{2}$ & Environment parameters & 0.14 \\
$\eta_{L o S}$ & Additional path loss for LoS & $3 \mathrm{~dB}$ \\
$\eta_{N L o S}$ & Additional path loss for NLoS & $23 \mathrm{~dB}$ \\
$\alpha$ & Path loss exponent & 2 \\
$B$ & Total Bandwidth of UAV m & $10 \mathrm{MHz}$ \\
$F$ & Scaling factor & 0.6 \\
$C R$ & Crossover factor & 0.5 \\
\hline \hline
\end{tabular}

\section{B. Simulation Results Analysis}

Fig. 2 shows the result of 100 ground users associated with 5 UAVs. Ground users in the same cluster marked with the same color and symbol. It can be seen that the number of users in each cluster is equal, which verifies that the proposed algorithm can ensure the load balance. 


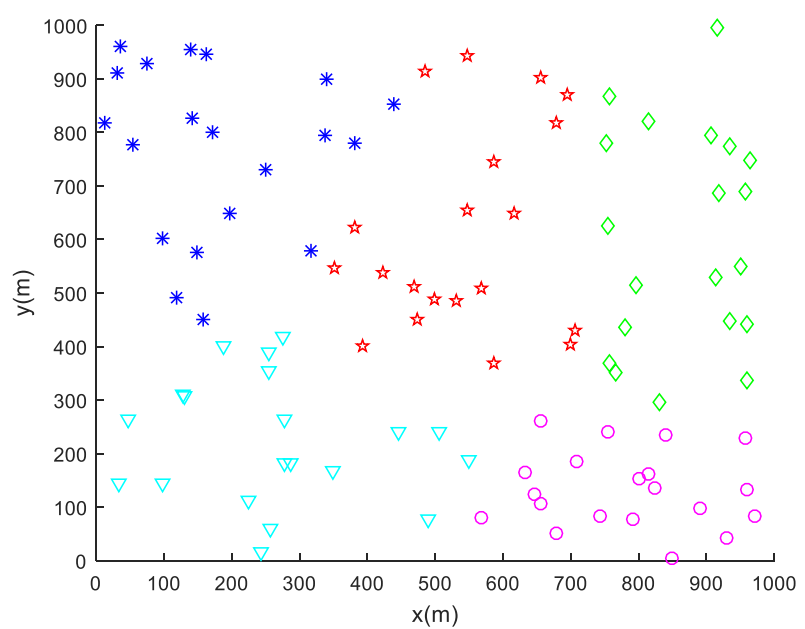

Fig.2 Balanced user clustering of 100 users

In order to show the effectiveness of the proposed algorithm, four different methods are compared: 'JHB' is joint height optimization and bandwidth allocation algorithm, in which the height is determined by using one dimension golden section search algorithm, and user association and UAVs' 2D horizontal location are determined by K-means++ algorithm. In 'Algorithm in [8]', UAVs' height depends on the user farthest away from the UAV and the fixed beam angle, and average bandwidth allocation. 'FLAB' refers to the fixed location of UAVs and average bandwidth allocation algorithm. All results are the average statistical results after 100 times independent simulations.

Fig. 3 shows the relation of the total transmit power of UAVs with the number of UAVs. we set 100 ground users randomly distributed in the square area, and $R_{k}^{\min }=3 \mathrm{Mbps}$.

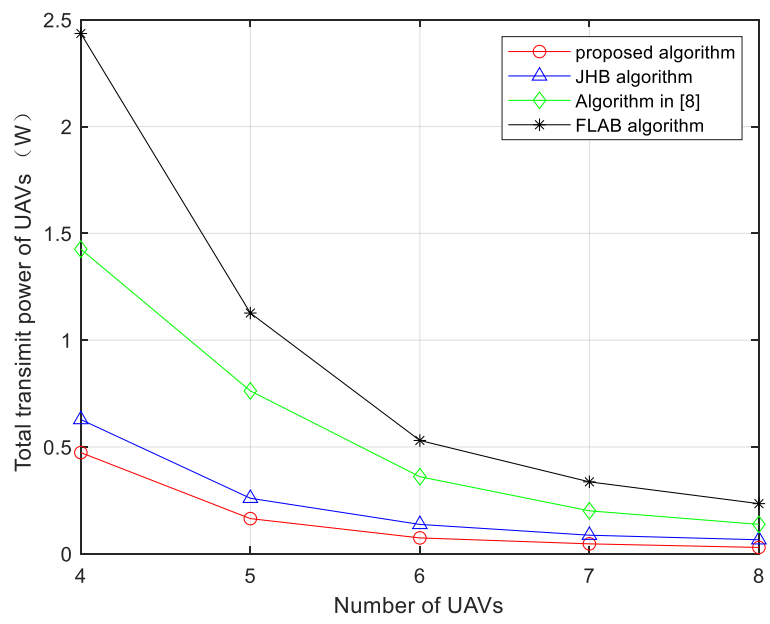

Fig. 3 Total transmit power for different numbers of UAVs

The total transmit power required by UAVs decreases with the increase of the number of UAVs. This is because when the number of users is fixed, the more UAVs are deployed, the shorter the average distance between UAVs and users, so each UAV requires smaller transmit power. And when the number of associated users of each UAV decreases, the average bandwidth available to each user increases, so the transmit power required to satisfy the minimum demand rate decreases.

It can be seen that the proposed algorithm outperforms other three algorithms. For JHB algorithm, it only optimize the height of UAVs rather than 3D deployment of UAVs, and it doesn't consider balanced user association. Although the algorithm in [8] optimizes the height, it does not optimize the bandwidth allocation. The performance of 'FLAB' algorithm is the worst due to fixed location and equal bandwidth allocation.

However, with the increase of the number of UAVs, the performance gap of various algorithms becomes smaller. The deployment of more UAVs will not bring more performance gain, but will increase the cost. Therefore, the deployment of an appropriate number of UAVs is also a key factor to be considered in system design, and it can be considered in future research.

Fig.4 shows the relation of the total transmit power required by UAVs with the minimum rate of users.

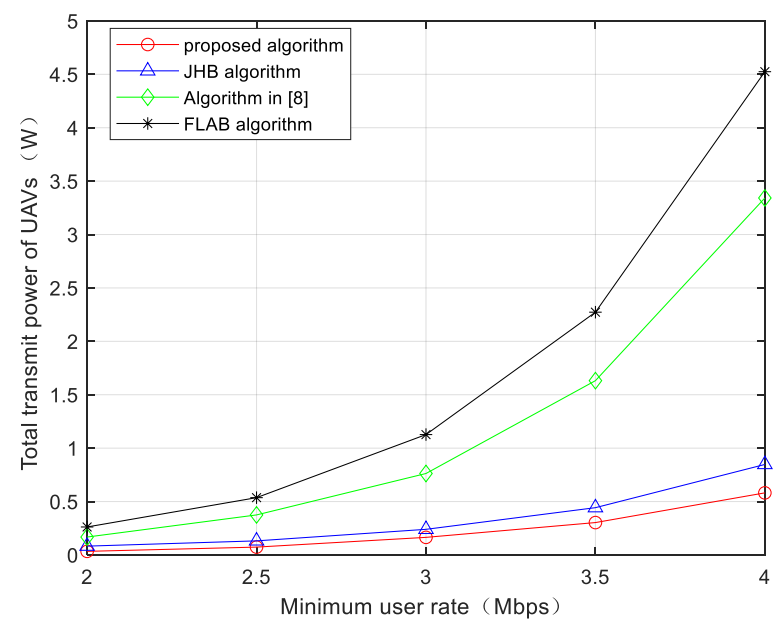

Fig. 4 Total transmit power for different minimum rate of users

The transmit power required by UAVs increases with the increasing demand rate of ground users. Simulation results show that the proposed algorithm outperforms other algorithms, especially when the user demand rate is high, because the proposed algorithm jointly optimizes 3D location and bandwidth allocation. The performance of 'JHB' algorithm is close to that of the proposed algorithm. This is because the UAV location is placed at the user's barycenter. Although it is not the optimal location, it is a suboptimal method because it reduces the distance the average distance between the users and the UAVs. The algorithm in [8] adjusts the height linearly according to the distance of the farthest associated user, and the bandwidth is evenly allocated. The performance of 'FLAB' algorithm is the worst due to its fixed location and average bandwidth allocation. This is because with the increase of user demand rate, in order to satisfy the requirement of some far edge users, the transmit power of UAVs will increase exponentially, and the farthest users and the closest users allocate the same bandwidth, so that the farther users need more transmit power to meet their minimum rate requirements. 


\section{CONCLUSION}

In this paper, the problem of 3D deployment optimization and bandwidth allocation in power efficient multi-UAV networks are investigated. With the constraints of meeting the minimum user demand rate and system load balancing, the total transmit power required by UAVs is minimized. The problem is decomposed into three sub-problems, and then solved by alternate iteration algorithm. Our proposed algorithm can be decomposed into three parts. First, to balance users associated to UAV, we use a balanced K-means clustering algorithm to divide all devices into several clusters, such that each cluster has approximately the same number of devices served by the same UAV. Then, we proposed a modified differential evolution algorithm to optimize the 3D deployment of multiUAVs to efficiently minimize total transmit power of UAVs. Finally, we obtain optimal bandwidth allocation by using convex optimization method. Simulation results confirm that the proposed algorithm that the total transmit power of UAVs significantly decreases compared with other methods.

\section{Acknowledgment}

This work is supported by the National Natural Science Foundation of China under Grant 62171345.

\section{REFERENCES}

[1] Zeng, Yong, Rui Zhang, and Teng Joon Lim. "Wireless communications with unmanned aerial vehicles: Opportunities and challenges."IEEE Communications Magazine 54.5 (2016): 36-42.

[2] Mozaffari, Mohammad, et al. "A tutorial on UAVs for wireless networks: Applications, challenges, and open problems."IEEE communications surveys \& tutorials 21.3 (2019): 2334-2360.

[3] Chen, Mingzhe, et al. "Caching in the sky: Proactive deployment of cache-enabled unmanned aerial vehicles for optimized quality-ofexperience."IEEE Journal on Selected Areas in Communications 35.5 (2017): 1046-1061.

[4] Wang, Lei, Bo Hu, and Shanzhi Chen. "Energy efficient placement of a drone base station for minimum required transmit power."IEEE Wireless Communications Letters 9.12 (2018): 2010-2014.

[5] Sawalmeh, Ahmad, Noor Shamsiah Othman, and Hazim Shakhatreh "Efficient deployment of multi-UAVs in massively crowded events."Sensors 18.11 (2018): 3640.

[6] Mozaffari, Mohammad, et al. "Mobile unmanned aerial vehicles (UAVs) for energy-efficient Internet of Things communications."IEEE Transactions on Wireless Communications 16.11 (2017): 7574-7589.

[7] Mozaffari, Mohammad, et al. "Optimal transport theory for powerefficient deployment of unmanned aerial vehicles." 2016 IEEE international conference on communications (ICC). IEEE, 2016.

[8] Ei, Nway Nway, et al. "Cell association in energy-constrained unmanned aerial vehicle communications under altitude consideration." 2019 International Conference on Information Networking (ICOIN). IEEE, 2019.

[9] Liu, Yanming, et al. "Resource Allocation and 3D Placement for UAVEnabled Energy-Efficient IoT Communications."IEEE Internet of Things Journal (2020).

[10] Sun, Yang, Tianyu Wang, and Shaowei Wang. "Location optimization and user association for unmanned aerial vehicles assisted mobile networks."IEEE Transactions on Vehicular Technology 68.10 (2019): 10056-10065.

[11] Mozaffari, Mohammad, et al. "Wireless communication using unmanned aerial vehicles (UAVs): Optimal transport theory for hover time optimization."IEEE Transactions on Wireless Communications 16.12 (2017): 8052-8066.

[12] Sami, Mahmoud, and John N. Daigle. "User association and power control for UAV-enabled cellular networks."IEEE Wireless Communications Letters 9.3 (2019): 267-270.
[13] Yang, Lei, et al. "Multi-UAV-enabled load-balance mobile-edge computing for IoT networks."IEEE Internet of Things Journal 7.8 (2020): 6898-6908.

[14] Yang, Helin, and Xianzhong Xie. "Energy-efficient joint scheduling and resource management for UAV-enabled multicell networks."IEEE Systems Journal 14.1 (2019): 363-374.

[15] Al-Hourani, Akram, Sithamparanathan Kandeepan, and Simon Lardner. "Optimal LAP altitude for maximum coverage."IEEE Wireless Communications Letters 3.6 (2014): 569-572.

[16] Malinen, Mikko I., and Pasi Fränti. "Balanced k-means for clustering."Joint IAPR International Workshops on Statistical Techniques in Pattern Recognition (SPR) and Structural and Syntactic Pattern Recognition (SSPR). Springer, Berlin, Heidelberg, 2014.

[17] Wang, Yong, et al. "Differential evolution with a new encoding mechanism for optimizing wind farm layout."IEEE Transactions on Industrial Informatics 14.3 (2017): 1040-1054.

[18] Yang, Zhaohui, et al. "Joint time allocation and power control in multicell networks with load coupling: Energy saving and rate improvement."IEEE Transactions on Vehicular Technology 66.11 (2017): 10470-10485.

The authors declear that they have no known competing financial interests or personal relationships that could have apperared to influence the work reported in this paper. 